\title{
Recuperación estructural en bosques sucesionales andinos de Porce (Antioquia, Colombia)
}

\author{
Adriana P. Yepes ${ }^{1}$, Jorge I. del Valle ${ }^{2}$, Sandra L. Jaramillo ${ }^{3} \&$ Sergio A. Orrego ${ }^{2}$ \\ 1. Centro de Investigación en Ecosistemas y Cambio Global-Carbono \& Bosques. Calle51A No 72-23, interior 601, \\ Medellín, Colombia; adrianayepes@carbonoybosques.org \\ 2. Departamento de Ciencias Forestales, Universidad Nacional de Colombia, A.A. 1779, Medellín, Colombia; \\ jidvalle@unal.edu.co,saorrego@unal.edu.co \\ 3. Universidad Nacional de Colombia, A.A. 1779, Medellín, Colombia; sljarami@unal.edu.co
}

Recibido 02-III-2009. C Corregido 15-VIII-2009. Aceptado 16-IX-2009.

\begin{abstract}
Structural recovering in Andean successional forests from Porce (Antioquia, Colombia). Places subjected to natural or human disturbance can recover forest through an ecological process called secondary succession. Tropical succession is affected by factors such as disturbances, distance from original forest, surface configuration and local climate. These factors determine the composition of species and the time trend of the succession itself. We studied succession in soils used for cattle ranching over various decades in the Porce Region of Colombia (Andean Colombian forests). A set of twenty five permanent plots was measured, including nine plots $(20 \times 50 \mathrm{~m})$ in primary forests and sixteen $(20 \times 25 \mathrm{~m})$ in secondary forests. All trees with diameter $\geq 1.0 \mathrm{~cm}$ were measured. We analyzed stem density, basal area, above-ground biomass and species richness, in a successional process of $c a$. 43 years, and in primary forests. The secondary forests' age was estimated in previous studies, using radiocarbon dating, aerial photographs and a high-resolution satellite image analysis ( 7 to $>43$ years). In total, 1143 and 1766 stems were measured in primary and secondary forests, respectively. Basal area $\left(5.7\right.$ to $\left.85.4 \mathrm{~m}^{2} \mathrm{ha}^{-1}\right)$, above-ground biomass (19.1 to $1011.5 \mathrm{t} \mathrm{ha}^{-1}$ ) and species richness (4 to 69) directly increased with site age, while steam density decreased (3 180 to 590). Diametric distributions were "J-inverted" for primary forests and even-aged size-class structures for secondary forests. Three species of palms were abundant and exclusive in old secondary forests and primary forests: Oenocarpus mapora, Euterpe precatoria and Oenocarpus bataua. These palms happened in cohorts after forest disturbances. Secondary forest structure was $40 \%$ in more than 43 years of forest succession and indicate that many factors are interacting and affecting the forests succession in the area (e.g. agriculture, cattle ranching, mining, etc.). Rev. Biol. Trop. 58 (1): 427-445. Epub 2010 March 01.
\end{abstract}

Key words: above-ground biomass, diametric structure, primary forests, secondary forests, vegetal succession.

La sucesión vegetal secundaria es un proceso ecológico por el cual se recupera la cobertura boscosa en lugares que anteriormente estuvieron sometidos a algún tipo de perturbación natural o antrópica. En general, la sucesión secundaria presenta rasgos comunes en las regiones tropicales, pero varía de una localidad a otra dependiendo de las condiciones del sitio como tipo e intensidad de perturbación, distancia al bosque original, fauna, topografía y clima local que determinan la velocidad con la que el bosque se recupera (Saldarriaga 1991, Finegan 1996, 1997, Guariguata \& Ostertag 2001, Kennard 2001). En el trópico algunos autores reportan que la riqueza, composición florística y las características estructurales como la distribución diamétrica, el área basal y la biomasa aérea, pueden llegar a parecerse a las del bosque primario durante los primeros 30 a 50 años de la sucesión (Ewel 1980, Brown \& Lugo 
1990, Ferreira \& Prance 1999, De Walt et al. 2003) o por el contrario, diferir enormemente por lo menos durante un siglo, dependiendo de la intensidad del uso previo del suelo (Finegan 1996, Ferreira \& Prance 1999, Guariguata \& Ostertag 2001, De Walt et al. 2003). Dado que los bosques secundarios tienen gran importancia ecológica porque proveen numerosos bienes y servicios ambientales, entre los cuales pueden mencionarse la producción de leña y carbón vegetal, protección de la fauna y de la flora, control de la erosión, regulación hídrica, captura de carbono, entre otros (Phillips \& Gentry 1994, Guariguata \& Ostertag 2001, Clark 2007), conocer la trayectoria sucesional que los genera es importante para planificar su uso, manejo y conservación.

Durante décadas los bosques de la región de Porce (Antioquia, Colombia) han sido talados y quemados para establecer pasturas ganaderas y, en menor proporción agricultura. Este tipo de prácticas pudieron ser decisivas para el proceso sucesional del sitio, ya que la degradación producida en el suelo pudo afectar las características estructurales de la vegetación secundaria establecida (Aide et al. 1995, Finegan 1996, Ferreira \& Prance 1999). Los resultados de investigaciones preliminares en esta región, sugieren la existencia de una sucesión degradada (Toro 2004, Yepes-Quintero et al. 2007), con claras evidencias de compactación de los suelos hasta $30 \mathrm{~cm}$ de profundidad (Lara 2003), gran dominancia de dos especies pioneras típicas de suelos empobrecidos del género Vismia y tasas de acumulación de biomasa menores en bosques secundarios en relación con la que presentan los primarios (Orrego \& del Valle 2003, Sierra et al. 2007). Este trabajo tuvo por objetivo analizar el área basal, la biomasa aérea, la riqueza de especies y la estructura diamétrica del proceso sucesional, en suelos dedicados previamente a la ganadería extensiva, desde la fase de bosques secundarios tempranos hasta fases más avanzadas de la sucesión, y compararlas con los bosques primarios cercanos.

\section{MATERIALES Y MÉTODOS}

Área de estudio: La investigación se llevó a cabo en la Región de Porce, Antioquia, Colombia $\left(6^{\circ} 45^{\prime} 37^{\prime \prime} \mathrm{N}, 75^{\circ} 06^{\prime} 28^{\prime}\right.$ ' W), localizada en el norte de la Cordillera Central de los Andes colombianos. La precipitación promedio anual entre 1999 y 2003 fue $2078 \pm 601 \mathrm{~mm}$ $( \pm \mathrm{DE})$ y es relativamente homogénea durante el año, presenta un período seco $(<15 \mathrm{~mm}$ por mes) entre diciembre y enero (Moreno 2004, Sierra et al. 2007). La temperatura promedio anual a $975 \mathrm{~m}$ de altitud es $22.7^{\circ} \mathrm{C}$, con promedio mínimo de $21.3^{\circ} \mathrm{C}$ y máximo de $24.1^{\circ} \mathrm{C}$. La elevación fluctúa entre 900 y $1500 \mathrm{~m}$ y la zona de vida corresponde a la transición entre los pisos tropical y premontano. La región está dominada por rocas ígneas intermedias del Cretáceo de tipo cuarzo-diorita y esquistos de composición clorítica. Los suelos son jóvenes, con baja fertilidad natural y buen drenaje (excepto en áreas plano-cóncavas). Veinte series de suelos han sido descritas y agrupadas en dos grandes órdenes: Entisoles e Inceptisoles. Los suelos más comunes son los subgrupos Ustoxic Dystropept, Typic Tropaquent y Typic Tropopsamment (Jaramillo 1989). En general, la densidad media aparente de éstos a los $30 \mathrm{~cm}$ de profundidad, en bosques primarios es de $1.1 \mathrm{mg} \mathrm{m}^{-3}$, mientras que en los secundarios es de $1.3 \mathrm{mg} \mathrm{m}^{-3}$ (Lara 2003).

En la región, evidencias de asentamientos humanos se remontan a 9000 años atrás, cuando se establecieron comunidades indígenas en las orillas del actual río Porce y la quebrada La Cancana (Castillo 1998). Después de la colonización española ( $\approx$ siglo XVII), el uso del suelo cambió a ganadería extensiva, minería y agricultura en pequeñas parcelas. Posteriormente, durante la década de 1990 los agricultores y ganaderos, vendieron o abandonaron las tierras lo que promovió la sucesión vegetal. Actualmente, en la región existe un mosaico de bosques primarios y bosques sucesionales. Los fragmentos de bosques primarios cubren cerca de 694ha y los bosques secundarios $1462 \mathrm{ha}$ 
(Orrego \& del Valle 2003). La composición y diversidad de especies en estos sitios es similar a la reportada para otros bosques tropicales de tierras bajas. Las principales especies de bosque primario según el índice de valor de importancia son: Anacardium excelsum (Bertero and Balb. ex Kunth), Jacaranda copaia (Aubl.) D. Don, Pourouma cecropiifolia Mart., Virola sebifera Aubl., Oenocarpus bataua Mart., Miconia albicans (Sw.) Triana, Vochysia ferruginea Mart., Cordia bicolor A. DC., Pera arborea Mutis y Pseudolmedia laevigata (Poepp. and Endl.) Rusby. En los bosques secundarios las especies dominantes son Vismia baccifera (L.) Triana y Planch y Piper aduncum L., seguidas por Myrsine guianensis A. DC., Jacaranda copaia, Psidium guajaba L., Miconia affinis DC., Erytroxylon sp., y Vismia ferruginea H.B.K. (Yepes-Quintero et al. 2007).

Colección de datos: Durante 1999 y 2000, se establecieron de manera aleatoria, 32 parcelas permanentes $(20 \times 50 \mathrm{~m})$ en los bosques primarios y 90 parcelas permanentes $(20 \times 25 \mathrm{~m})$ en los bosques secundarios de la Región de Porce (Sierra et al. 2007). En los bosques primarios el tamaño de las parcelas fue de $1000 \mathrm{~m}^{2}$ (50x20m). En ellas se midió el diámetro normal (D) a $1.3 \mathrm{~m}$ sobre el suelo o arriba de las bambas, si las hubiera, de todas las plantas leñosas (árboles, arbustos, palmas y lianas), con $\mathrm{D} \geq 10 \mathrm{~cm}$. Por lo regular el instrumento para esta medición fue el calibrador forestal (forcípula) con exactitud de $0.5 \mathrm{~mm}$. Además, en el tercer vértice de cada parcela se levantó una subparcela de 0.01 ha $(10 \times 10 \mathrm{~m})$, en la cual se midieron con calibrador digital, con exactitud de $0.01 \mathrm{~mm}$, todas las plantas leñosas con $1 \mathrm{~cm} \leq \mathrm{D}<10 \mathrm{~cm}$. En los bosques secundarios se aplicó un protocolo similar, excepto que las parcelas fueron de $500 \mathrm{~m}^{2}(25 \times 20 \mathrm{~m})$ y las subparcelas de 0.0025 ha $(5 \times 5 \mathrm{~m})$. En todas las parcelas se midieron las plantas leñosas con $\mathrm{D} \geq 5 \mathrm{~cm}$, y aquellas con $1 \mathrm{~cm} \leq \mathrm{D}<5 \mathrm{~cm}$ se midieron en las subparcelas.

En el caso de las palmas, se aplicó un método de medición diferente que permitiera posteriormente determinar su biomasa y tasa de crecimiento. Generalmente, los monitoreos de biomasa y carbono han sido diseñados o asesorados por científicos fuera del trópico, donde las palmas son inexistentes o muy escasas, esto ha llevado a que no se propongan mediciones especiales para ellas (Brown 1997, MacDicken 1997, Márquez \& Roy 2000). De esta manera, al monitorear el crecimiento diamétrico de las palmas, su tasa de cambio es cero por cuanto estas plantas no presentan crecimiento secundario (diamétrico) notorio como en el caso de los árboles. Este hecho ha ocasionado que se subvalore las tasas de captura de carbono por los bosques tropicales, donde las palmas constituyen un elemento florístico y estructural muy importante. A pesar de que algunos recomiendan ecuaciones específicas para las palmas (Márquez \& Roy 2000), no es claro cómo se monitorea el crecimiento en altura, dado que es bien conocido que la mayoría de los instrumentos empleados para medir esta variable tienen baja exactitud. Teniendo en cuenta estos antecedentes, y partiendo del hecho de que las palmas tienen un número constante de hojas durante su lapso vital y su crecimiento primario en altura es rítmico, se ascendió a ellas hasta el punto de inserción de la vaina de la última hoja (la más baja) en el estipe, y se midió con cinta métrica la longitud del estipe hasta ese punto. Luego se marcó con pintura amarilla tanto la última porción de la vaina de la hoja, cerca de su punto de inserción, como parte del estipe. Ello tuvo por finalidad, que al desprenderse esta hoja, quede marca amarilla circular alrededor del estipe, exactamente en el punto donde el desprendimiento de la hoja, deja una cicatriz natural. La distancia entre la base de esta cicatriz en el estipe, y la base de la hoja que quedó de última, registra el crecimiento en altura del estipe durante el tiempo transcurrido entre la formación de estas dos hojas. A cada palma también se le midió el diámetro en la base del estipe (Orrego \& del Valle 2003).

Para esta investigación, fueron seleccionadas veinticinco parcelas al azar: nueve en bosques primarios y dieciséis en bosques secundarios. En las parcelas seleccionadas se realizó la identificación botánica de las 
especies siguiendo los protocolos convencionales (Ricker \& Daly 1998), y cada individuo fue clasificado en una categoría o hábito de crecimiento de acuerdo con sus características ecofisiológicas. Las categorías fueron: árbol, arbusto, hierba, helecho, palma y liana. Una completa descripción de la colección de datos florísticos se encuentra en Yepes-Quintero et al. (2007). Los datos estructurales empleados en esta investigación corresponden a la medición realizada durante el período junio-julio de 2003 en el marco del proyecto "Sucesión vegetal, diversidad biológica y acumulación de carbono en el cañón del río Porce, Antioquia" (Orrego \& del Valle 2003).

Determinación de la edad de las parcelas de bosques secundarios: La edad de 12 parcelas de bosque secundario se obtuvo con datación por carbono catorce $\left(\mathrm{C}^{14}\right)$ por ciento moderno, método radiométrico basado en la vida media del isótopo $\mathrm{C}^{14}$ y comúnmente empleado para determinar la edad de materiales orgánicos portadores de carbono, cuya antigüedad esté comprendida entre el presente (P) y 40 000-50 000 años atrás. Para la determinación de la edad por el método del $\mathrm{C}^{14}$ se partió de la hipótesis de que los árboles de estos procesos sucesionales tempranos son en esencia coetáneos (Finegan 1996, 1997). De esta manera, se calculó el diámetro promedio para cada una de las parcelas seleccionadas, y posteriormente se seleccionó un árbol con diámetro similar por fuera de ellas, para el muestreo. Cada árbol fue cosechado y se extrajo una sección transversal de la base del fuste con una sierra manual, con el fin de evitar la contaminación con el carbono del aceite de la cadena si se hubiese usado una motosierra. Del centro anatómico de cada sección transversal, que contiene la madera más antigua del árbol, se extrajo con un taladro unos $15-25 \mathrm{~g}$ de madera seca, que posteriormente se enviaron al laboratorio Beta Analytic en Estados Unidos para la determinación del $\% \mathrm{C}^{14}$ moderno (Posterior a 1950, fecha denominada como $\mathrm{P}$ o presente) por el método radiométrico convencional. La datación de los árboles por este sistema hace uso del llamado efecto de las bombas nucleares en la concentración de radiocarbono en el $\mathrm{CO}_{2}$ atmosférico durante la década de los 50's y principios del 60, el cual produjo cantidades adicionales de $\mathrm{C}^{14}$ de las normalmente producidas por los rayos cósmicos. $\mathrm{El} \mathrm{C}^{14}$ una vez oxidado en $\mathrm{CO}_{2}$ es tomado por las plantas y transformado en madera, constituyendo un marcador isotópico permanente: concentración del $\mathrm{C}^{14}$ en el $\mathrm{CO}_{2}$ atmosférico $\approx$ concentración del $\mathrm{C}^{14}$ en la madera (Worbes 1989). Los resultados de radiocarbono se calibraron mediante el programa Calibomb (Hua \& Barbetti 2004, Reimer et al. 2004) para la zona 2 correspondiente a los trópicos del Hemisferio Norte de acceso libre en la página en la revista Radiocarbon. Este programa permite determinar con un alto grado de confiabilidad el año de formación de la madera y, por tanto, la edad del árbol: año en que se cortó el árbol menos el año de formación de la primera madera. De esta manera, la edad promedio de los bosques secundarios se interpretó como la edad del árbol con diámetro promedio muestreado (Sierra et al. 2007).

Para las cuatro parcelas restantes, la edad se determinó utilizando secuencias temporales de fotografías aéreas de 1961, 1979 y 1989, y una sub-escena Ikonos de 2001. El procedimiento consistió en primer lugar, en fotointerpretar estereoscópicamente las fotografías aéreas y clasificar la imagen mediante correcciones y realces. Posteriormente, se separaron las coberturas vegetales con la imagen Ikonos discriminando: cultivos, pastos, pastos enrastrojados, vegetación secundaria (rastrojos bajos y altos), bosques secundarios y bosques primarios. Los cambios de cobertura vegetal identificados entre el período 1961 y 2001, permitieron identificar claramente la edad de estas cuatro parcelas, y corroborar las edades obtenidas con el método de $\mathrm{C}^{14}$ (Agudelo \& Restrepo 2004).

Estimación de la biomasa aérea: Para la estimación de la biomasa aérea se cosecharon y midieron árboles, palmas y lianas. Para evitar extrapolaciones al momento de predecir la biomasa de grandes individuos, se 
seleccionaron árboles de todos los tamaños (D entre 0.3 y $198.9 \mathrm{~cm}$ ). El número total de árboles cosechados fue de 292, y a cada uno de ellos se les midió el diámetro y la longitud del fuste con cinta métrica. Para encontrar la masa verde de cada árbol se trabajó por componentes (hojas, ramitas con $\mathrm{D}<2.5 \mathrm{~cm}$, ramas y fuste). Los árboles con $\mathrm{D}>30 \mathrm{~cm}$ se cubicaron. El peso seco de los elementos cubicados se encontró posteriormente con base en muestras tomadas para la determinación de la densidad básica. El peso seco de los componentes de cada árbol y el contenido de humedad de los mismos, se determinó a partir de muestras de $500 \mathrm{~g}$ tomadas en campo. Este material se secó en un horno a $103^{\circ} \pm 1^{\circ} \mathrm{C}$ hasta alcanzar peso constante. La masa seca de cada componente por árbol, se determinó con la relación masa seca a masa verde. Finalmente, la masa seca total de cada árbol se obtuvo al sumar todas las masas secas de los componentes. Un procedimiento similar se llevó a cabo para las palmas y lianas. En el caso de las palmas, 120 individuos fueron cosechados (83 de Oenocarpus bataua Mart., 15 de Oenocarpus mapora H. Karst., 12 de Euterpe precatoria Mart. y 10 Bactris sp.). A partir de estos datos se construyeron las ecuaciones de biomasa locales que se presentan en el Cuadro 1. Mayores detalles sobre el ajuste de los modelos se encuentra en Zapata et al. (2003). Estas ecuaciones fueron empleadas para calcular la biomasa de cada individuo. La biomasa aérea total de cada parcela, se calculó como la sumatoria de la biomasa aérea de todos los árboles, palmas y lianas.

Análisis de datos: Para evaluar si existían diferencias entre el número de árboles por hectárea, el área basal, la biomasa aérea y la riqueza de especies, se realizó en primer lugar, una prueba Shapiro-Wilk con el fin de verificar la normalidad de los datos (Walpole \& Myers 1987, Sprent 1989). Debido a que ningún conjunto de datos presentó distribución Normal, se utilizó la prueba no paramétrica Kruskal-Wallis (H) para inferir la existencia o no de diferencias a lo largo del proceso sucesional respecto a estas características. Cuando se encontraron diferencias significativas, se empleó adicionalmente, la prueba de Rangos Múltiples de Bonferroni $(\alpha=0.05)$ para evaluar cuáles medias eran significativamente diferentes de otras (Walpole \& Myers 1987). Adicionalmente, con el objetivo de representar la tendencia sucesional de cada variable, se ajustó una regresión lineal simple utilizando el método de mínimos cuadrados ordinarios para calcular la línea recta que mejor representara el comportamiento de los datos analizados (Walpole \& Myers 1987).

La estructura diamétrica se obtuvo para todos los árboles con $\mathrm{D} \geq 1.0 \mathrm{~cm}$ graficando histogramas de abundancia $v s$. clase de tamaño.

CUADRO 1

Ecuaciones de biomasa aérea construidas para el área de estudio, Región de Porce (Antioquia-Colombia)

TABLE 1

Above-ground Equations built for the study area, Porce Region (Antioquia-Colombia)

$\begin{array}{llccc}\text { Tipo de planta } & \text { Ecuación } & \mathrm{FC} & \mathrm{n} & \% \mathrm{R}^{2} \\ \text { Árboles del bosque primario }(\mathrm{D} \geq 1 \mathrm{~cm}) & \ln (\mathrm{BA})=-2.274+2.455 \ln (\mathrm{D}) & 0.091 & 140 & 97.90 \\ \text { Árboles del bosques secundarios }(\mathrm{D} \geq 1 \mathrm{~cm}) & \ln (\mathrm{BA})=-2.232+2.422 \ln (\mathrm{D}) & 0.083 & 152 & 97.47 \\ \text { O. bataua } & \mathrm{BA}=\mathrm{e}^{1.989} \mathrm{~L}^{1.133}+139.48 & - & 83 & 82.95 \\ \text { O. mapora } & \ln (\mathrm{BA})=-0.552+0.90 \ln (\mathrm{D}) & - & 15 & 89.10 \\ \text { E. precatoria } & \ln (\mathrm{BA})=-2.304+0.861 \ln \left(\mathrm{D}^{2} \mathrm{x} \mathrm{L}\right) & - & 12 & 98.36 \\ \text { Bactris sp. } & \ln (\mathrm{BA})=-5.559+0.08 \ln (\mathrm{L})+3.988 \ln (\mathrm{D}) & - & 10 & 88.51 \\ \text { Lianas } & \ln (\mathrm{BA})=0.028+1.841 \ln (\mathrm{D}) & 0.133 & 33 & 87.44\end{array}$

FC: factor de corrección (1/2 del cuadrado medio del error); $\mathbf{n}$ : tamaño de la muestra; $\mathbf{0} \mathbf{R}^{\mathbf{2}}$ : coeficiente de determinación en porcentaje; BA: Biomasa aérea en $\mathrm{kg}$; D: diámetro en $\mathrm{cm}$; L: longitud del estipe en $\mathrm{m}$. 
Se unificaron trece clases diamétricas para cada histograma con amplitud de $5.0 \mathrm{~cm}$ cada una, según el método propuesto por Silvertown \& Lovett Doust (1993). Los histogramas se construyeron para los bosques secundarios de las diferentes edades y los bosques primarios, con el fin de observar si el patrón estructural era el mismo. Posteriormente, la estructura diamétrica de algunas especies dominantes en bosques sucesionales avanzados, fue construida y analizada para inferir si su comportamiento condicionaba la estructura diamétrica total del bosque. De acuerdo con Rollet (1980), las especies tolerantes a la sombra o esciófitas presentan estructuras diamétricas en forma de $\mathrm{J}$ invertida, donde abundan los árboles pequeños. Por su parte, las especies demandantes de luz o heliófitas, presentan estructuras diamétricas unimodales o en forma de campana, en las que la mayoría de los árboles son coetáneos. Utilizando este planteamiento se pudo identificar el grupo ecológico de las especies analizadas. Finalmente, para determinar la estructura poblacional de las palmas se realizó un procedimiento análogo al de los histogramas de abundancia $v s$. clase de tamaño, pero empleando en lugar del diámetro, la variable altura.

\section{RESULTADOS}

Las parcelas de bosques secundarios conformaron una cronosecuencia con edades desde siete hasta más de cuarenta y tres años. Se muestrearon en total 2909 individuos de los cuales 1143 estuvieron en los bosques primarios y 1766 en los bosques secundarios. La densidad aparente de los suelos hasta $30 \mathrm{~cm}$ de profundidad, varió desde 1.08 hasta $1.60 \mathrm{Mgm}^{-}$ ${ }^{3} \mathrm{y}$, los valores más altos de esta característica, se presentaron en los bosques secundarios. Las especies más importantes en los bosques secundarios de acuerdo con lo reportado por YepesQuintero et al. (2007), fueron pioneras típicas como Vismia baccifera (L.) Triana y Planch, Piper aduncum L., y Psidium guajava L. Mientras que en los bosques primarios las especies más representativas fueron Anacardium excelsum (Bertero and Balb. ex Kunth), Jacaranda copaia (Aubl.) D. Don, Pourouma cecropiaefolia Mart., Oenocarpus bataua Mart., Vochysia ferruginea Mart., entre otras. La caracterización detallada de las 25 parcelas seleccionadas, se presenta en el Cuadro 2.

Estructura del bosque $y$ riqueza de especies: En general, el número de árboles disminuyó al incrementar la edad de la sucesión (Fig. 1A), y aunque la densidad de árboles fue variable a lo largo de todo el proceso sucesional, no se presentaron diferencias significativas entre los estadios sucesionales $(\mathrm{n}=6, \mathrm{H}=8.95, \mathrm{p}=0.06)$. Al evaluar el número de árboles discriminando por categoría diamétrica, este mismo patrón se obtuvo para los árboles con $1 \mathrm{~cm} \leq \mathrm{D}<10 \mathrm{~cm}(\mathrm{n}=6, \mathrm{H}=3.68$, $\mathrm{p}=0.45$ ). Sin embargo, para los árboles con $\mathrm{D} \geq 10 \mathrm{~cm}$, si se presentaron diferencias significativas $(\mathrm{n}=6, \mathrm{H}=17.24, \mathrm{p}=0.0018)$, siendo notorias las presentadas entre los bosques sucesionales tempranos (7-11 años) e intermedios (20-25 años), con los bosques sucesionales avanzados ( $>40$ años) y los bosques primarios (Bonferroni, $\mathrm{p}<0.05$; Fig. 1B). En términos de área basal, también se presentaron diferencias significativas $(n=6, H=18.09, p=0.0012)$, especialmente entre los bosques secundarios tempranos (7-11 años) y los bosques primarios (Bonferroni, $\mathrm{p}<0.05$; Fig. 1C). El área basal tendió al aumento a lo largo de la cronosecuencia estudiada, y los valores totales oscilaron entre 5.7 y $29.7 \mathrm{~m}^{2} \mathrm{ha}^{-1}$ para los bosques de 7 a 11 años, 25.2 y $35.5 \mathrm{~m}^{2} \mathrm{ha}^{-1}$ para los bosques de 25 a 40 años y, entre 24.3 y $85.4 \mathrm{~m}^{2} \mathrm{ha}^{-1}$ para los bosques primarios. La recuperación de esta característica alcanzó 47\% del valor encontrado para los bosques primarios.

Respecto a la biomasa aérea total por hectárea, ésta alcanzó valores desde 19.1 a 124.3 $\mathrm{t} \mathrm{ha}^{-1}$ para los bosques sucesionales tempranos (7-25 años), $218.6 \mathrm{t} \mathrm{ha}^{-1}$ para el bosque secundario de 40 años, y de 124.6 a $1011.5 \mathrm{t} \mathrm{ha}^{-1}$ para los bosques primarios. En los bosques primarios la biomasa aérea promedió $319.0 \mathrm{t} \mathrm{ha}^{-1}$, distribuida $92.66 \%$ en árboles y arbustos dicotiledones, $5.93 \%$ en palmas $(3.58 \%$ Oenocarpus bataua) y $1.41 \%$ en lianas. En el caso de los 


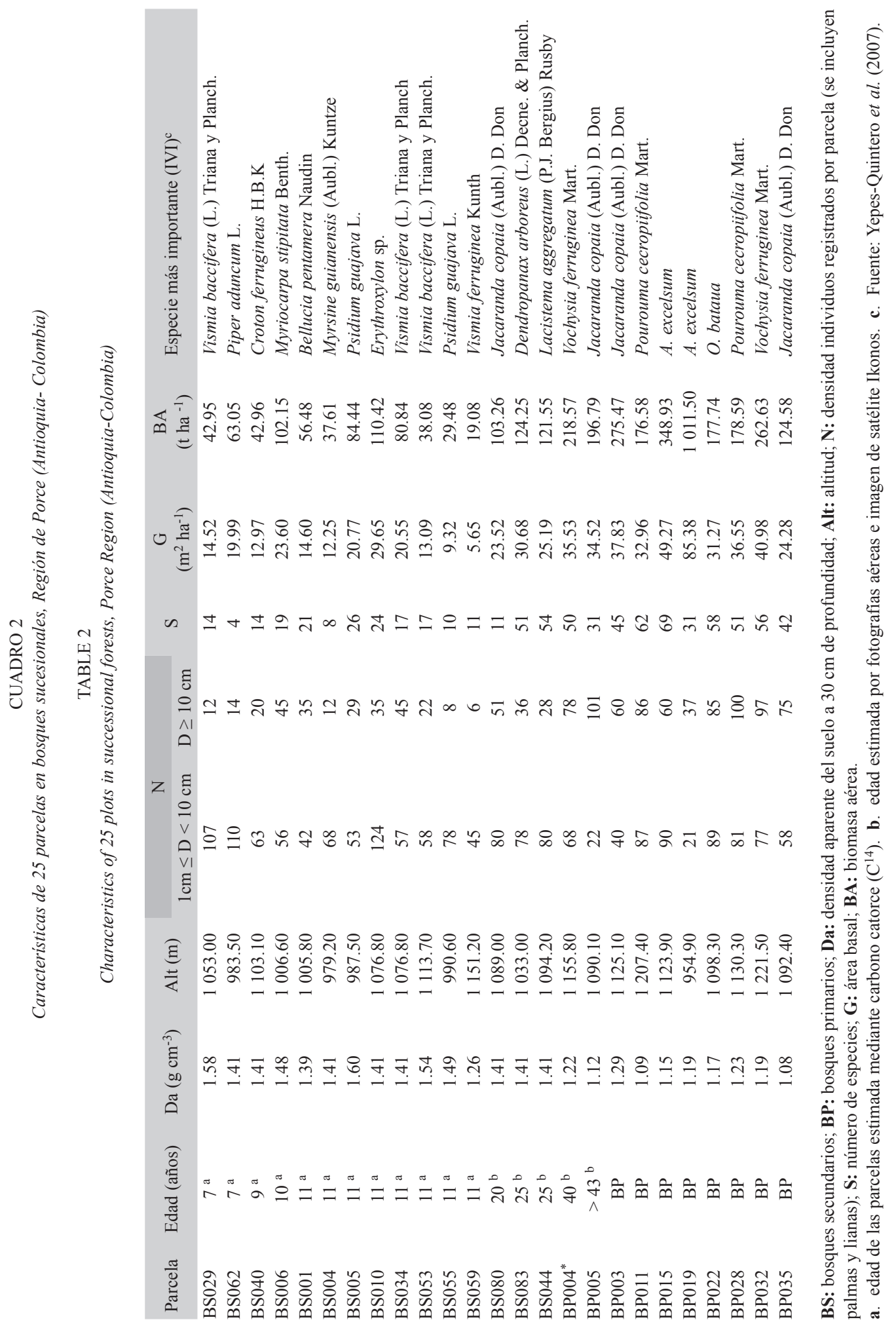



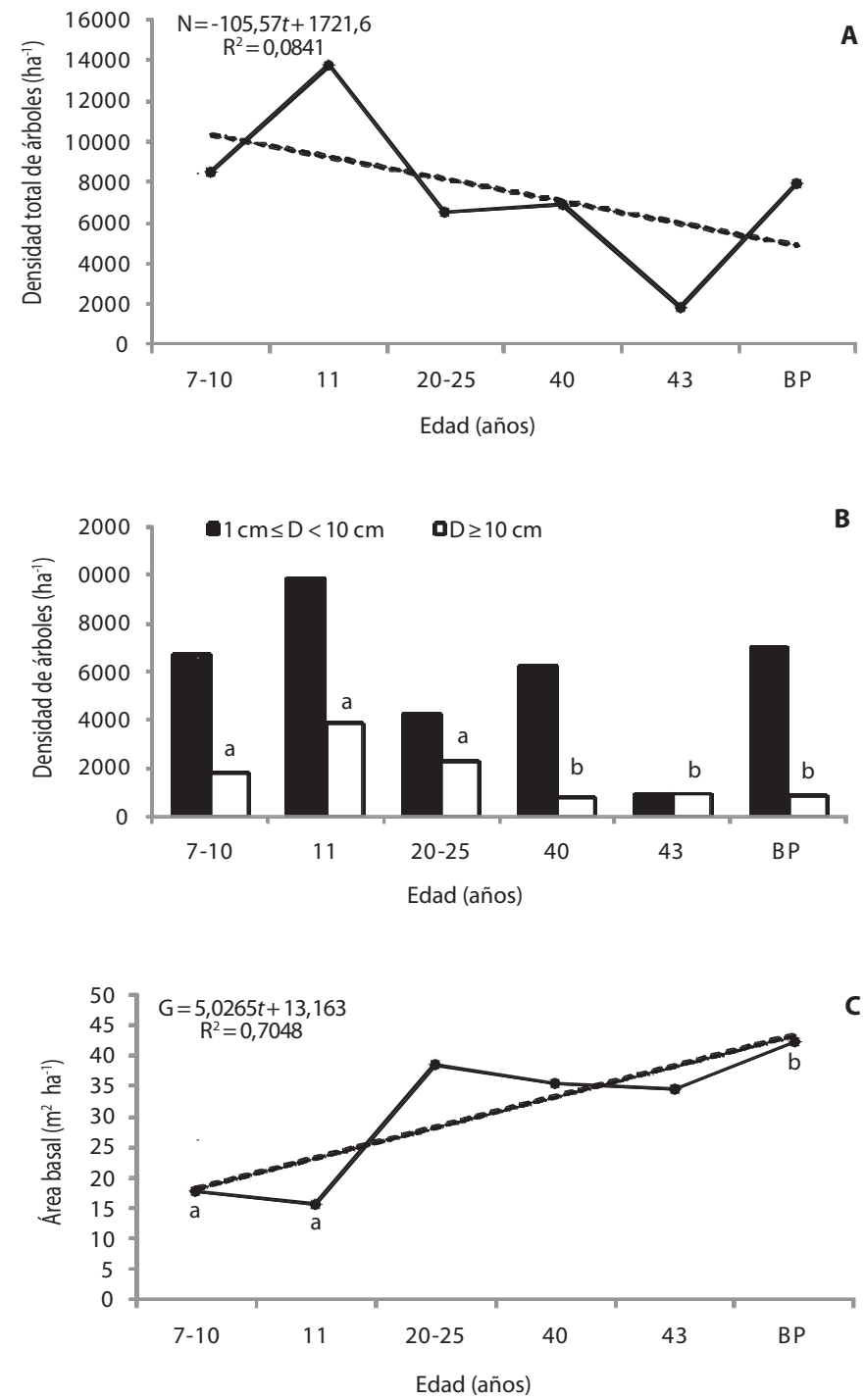

Fig. 1. (A) Densidad total de árboles por hectárea; (B) densidad de árboles por categoría diamétrica y (C) área basal, para individuos con $\mathrm{D} \geq 1.0 \mathrm{~cm}$. Líneas punteadas: tendencia del proceso sucesional (ecuación adjunta), las letras: diferencias significativas según la prueba Bonferroni $(\alpha=0.05)$.

Fig. 1. (A) Steam density per hectare; (B) steam density per diametric category and (C) area basal, for trees with $D \geq 1.0$ $\mathrm{cm}$. The discontinuous lines: tendency of the successional process (attached equation), the letters: significant differences according to the Bonferroni test ( $\alpha=0.05)$.

bosques secundarios la biomasa aérea promedio fue dos veces inferior a la alcanzada por los bosques primarios (130.3 $\left.\mathrm{tha}^{-1}\right)$, con aportes de $61.09 \%$ por árboles y arbustos, $0.71 \%$ de palmas y $38.2 \%$ de lianas. En general, la biomasa aérea varió significativamente durante el proceso sucesional $(n=6, H=19.62, p=0.0006)$, con diferencias marcadas entre los bosques secundarios tempranos (7-11 años) y los bosques primarios (Bonferroni, $\mathrm{p}<0.05$; Fig. $2 \mathrm{~A}$ ). $\mathrm{Al}$ 

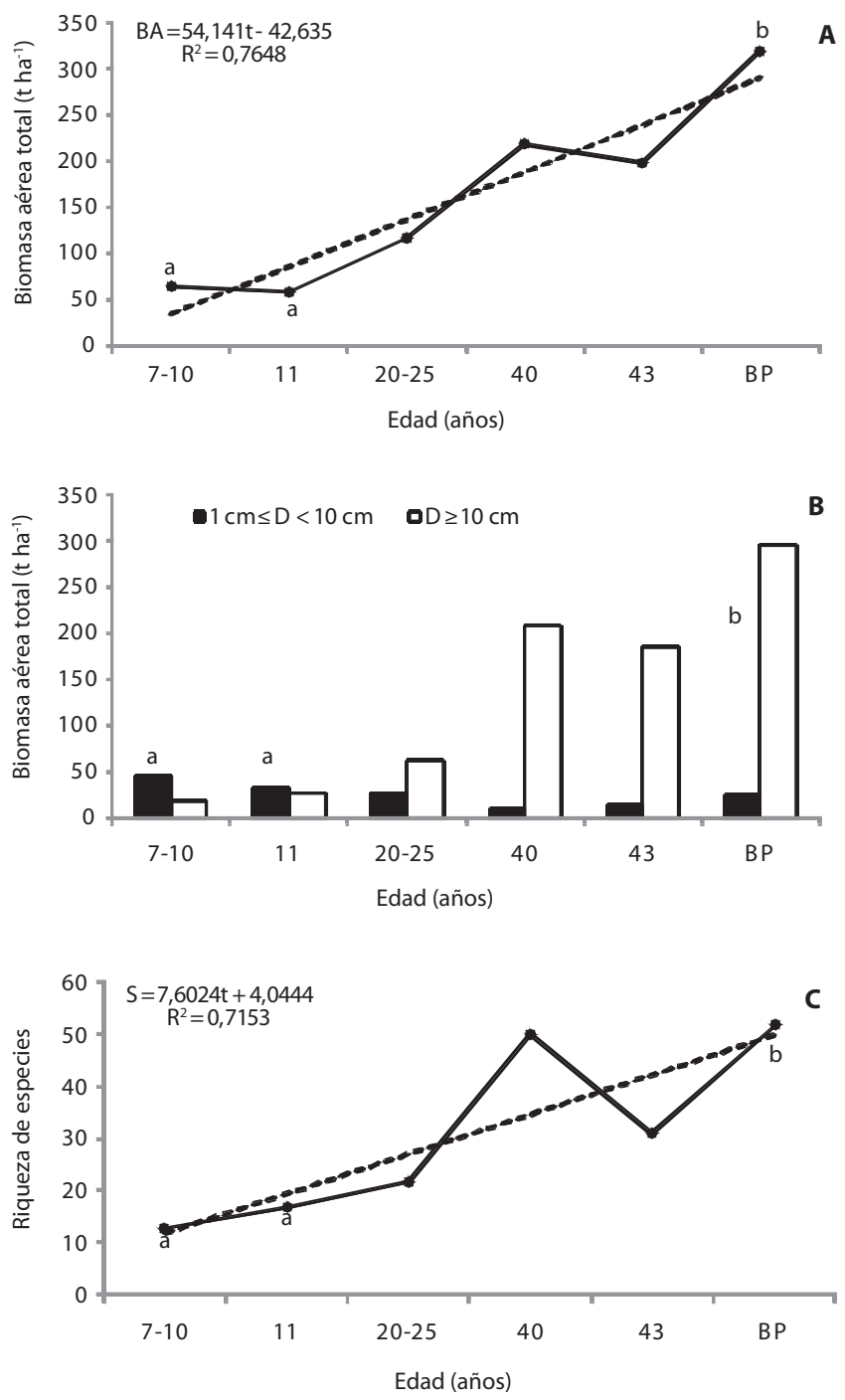

Fig. 2. (A) Biomasa aérea total en un proceso sucesional de c.a 43 años y en bosques primarios; (B) biomasa aérea por categoría diamétrica y $(\mathrm{C})$ riqueza de especies, para individuos con $\mathrm{D} \geq 1.0 \mathrm{~cm}$. Líneas punteadas: tendencia del proceso sucesional (ecuación adjunta), las letras: diferencias significativas según la prueba de Bonferroni $(\alpha=0,05)$.

Fig. 2. (A) Total above-ground biomass in a successional process of c.a. 43 years and primary forests; (B) above-ground biomass per diametric category and $(\mathrm{C})$ species richness, for trees with $\mathrm{D} \geq 1.0 \mathrm{~cm}$. The discontinuous lines: tendency of the successional process expressed (attached equation), the letters: significant differences according to the Bonferroni test $(\alpha=0.05)$.

igual que el área basal, la biomasa aérea total tendió a aumentar con el avance de la sucesión, aunque su recuperación promedio fue de $41 \%$, respecto al valor registrado para los bosques primarios. Finalmente, el mismo patrón se presentó cuando se analizó la biomasa aérea en términos de categorías diamétricas (Fig. 2B). En ambos casos las diferencias significativas se presentaron entre los bosques sucesionales tempranos (7-11 años) y los bosques primarios 
(Fig. 2B; $1 \mathrm{~cm} \leq \mathrm{D}<10 \mathrm{~cm}$ : $\mathrm{n}=6, \quad \mathrm{H}=12.60$, $\mathrm{p}=0.0134 ; \mathrm{D} \geq 10 \mathrm{~cm}: \mathrm{n}=6, \mathrm{H}=18.66, \mathrm{p}=0.0009$ ). Con relación a la riqueza de especies, esta característica aumentó gradualmente desde los estadios sucesionales tempranos hasta los más avanzados, presentando diferencias significativas entre ellos $(n=6, H=15.73, p=0.0034$; Fig. $3 \mathrm{C})$. El número promedio de especies en los bosques sucesionales alcanzó 44\% del valor presentado por los bosques primarios de la zona, cuando se igualaron las áreas de ambos tipo de bosque a $8000 \mathrm{~m}^{2}$.

Estructura diamétrica: Los histogramas de frecuencia muestran que los bosques secundarios de diferentes edades presentaron estructuras unimodales característica de comunidades coetáneas (Fig. 3A, B, C y E), a excepción del bosque de 40 años que presentó estructura en forma de $\mathrm{J}$ invertida (Fig. 3D). Este

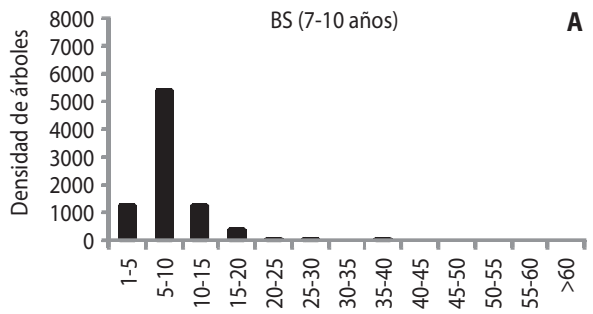

Clase diamétrica $(\mathrm{cm})$
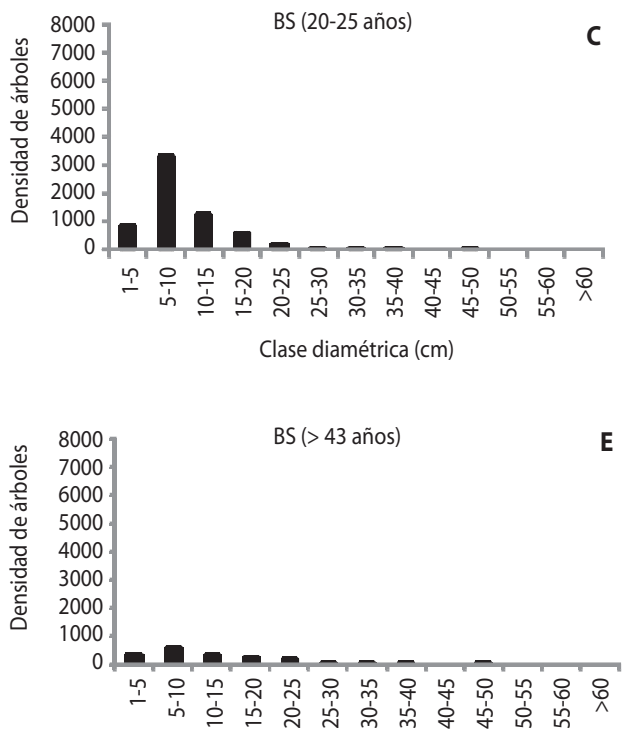

Clase diamétrica $(\mathrm{cm})$
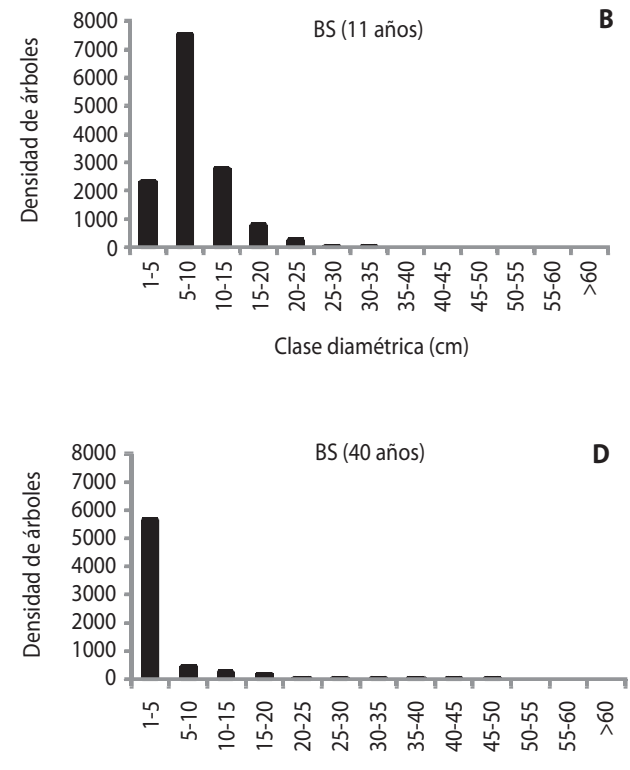

Clase diamétrica $(\mathrm{cm})$

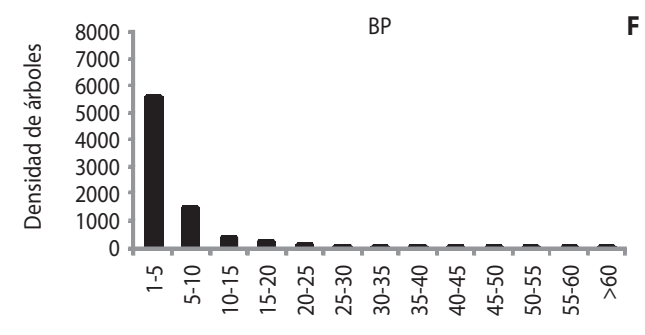

Clase diamétrica $(\mathrm{cm})$

Fig. 3. Estructura diamétrica en un proceso sucesional de c.a 43 años y bosques primarios. (A) bosques secundarios de 7 a 10 años; (B) bosques secundarios de 11 años; (C) bosques secundarios de 20 a 25 años; (D) bosque secundario de 40 años; (E) bosque secundario de $>43$ años y (F) bosques primarios. BS: bosques secundarios y BP: bosques primarios.

Fig. 3. Diametric structure in a successional process of c.a. 43 years and primary forests. (A) secondary forests from 7 to 10 years; (B) secondary forests of 11 years; (C) secondary forests from 20 to 25 years; (D) secondary forest of 40 years; (E) secondary forest of $>43$ years and $(\mathrm{F})$ primary forests. BS: secondary forest and BP: primary forests. 
comportamiento se explica por la abundante regeneración de Virola sebifera, una especie esciófita (según lo indica su distribución diamétrica), que aporta cantidades considerables de árboles pequeños $(1 \mathrm{~cm} \geq \mathrm{D}>5 \mathrm{~cm})$ a la primera clase diamétrica (Fig. 4A). Cuando dicha especie fue excluida de los análisis, la estructura diamétrica del bosque pasó a tener forma unimodal debido a que las demás especies abundantes, son especies heliófitas (Jacaranda copaia (Aubl.) D. Don., Enterolobium schomburgkii (Benth.) Benth., Vochysia ferruginea Mart., y Schefflera morototoni (Aubl.) Maguire, Steyerm. \& Frodin.), que presentan individual y conjuntamente distribuciones diamétricas de este tipo (Fig. 4B). Un patrón similar se observó para el bosque con más de 43 años de edad, donde abundan especies heliófitas de larga vida como Jacaranda copaia, Miconia albicans y Cordia bicolor (Fig. 3E). La estructura diamétrica de los bosques primarios presentó forma de $\mathrm{J}$ invertida, típica de comunidades disetáneas e irregulares (Fig. 3F).

Estructura para palmas: Las tres especies de palmas más abundantes en el área de estudio fueron Oenocarpus mapora, Euterpe precatoria y Oenocarpus bataua. En general la estructura por clases de alturas presentó para todos los casos comportamiento polimodal (Fig. 5A). Particularmente la estructura para Oenocarpus bataua tuvo tendencia irregular con dos picos evidentes, que sugieren la presencia de dos cohortes por clases de altura (Fig. 5B). Las palmas Oenocarpus mapora y Euterpe precatoria, presentaron también estructuras
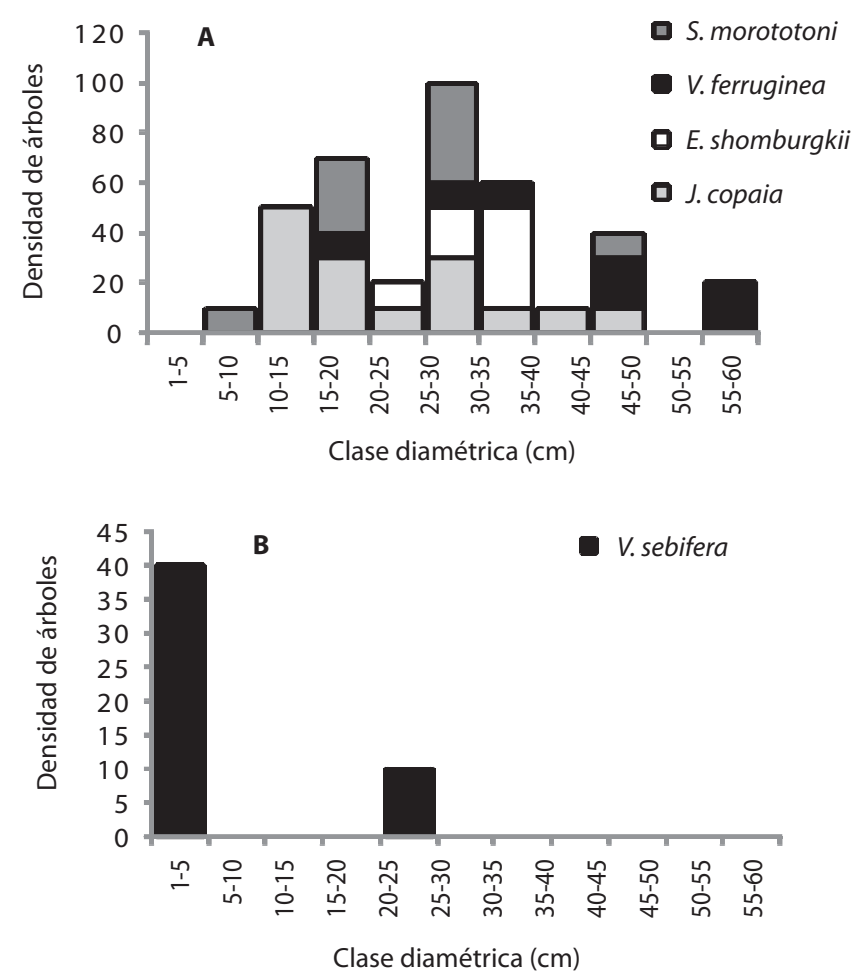

Fig. 4. Estructura diamétrica para las especies más abundantes en el bosque secundario de 40 años. (A) especies heliófitas y (B) especie esciófita.

Fig. 4. Diametric structure for the most abundant species in the secondary forest of 40 years. (A) light demanding species and (B) shadow tolerant specie. 


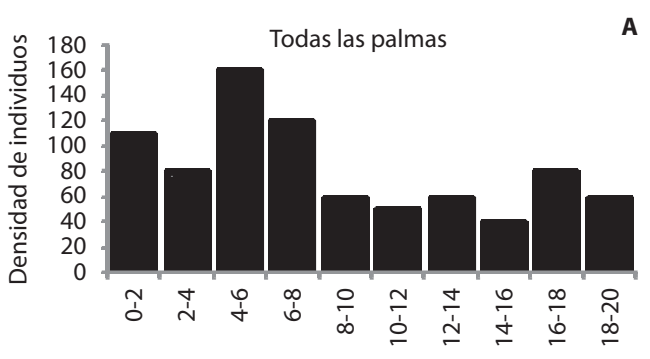

Clase de altura (m)

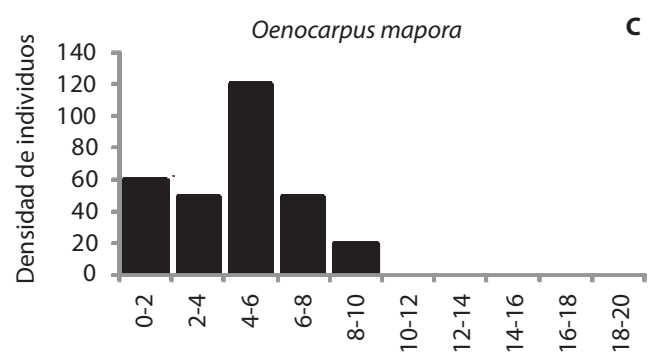

Clase de altura $(\mathrm{m})$

A
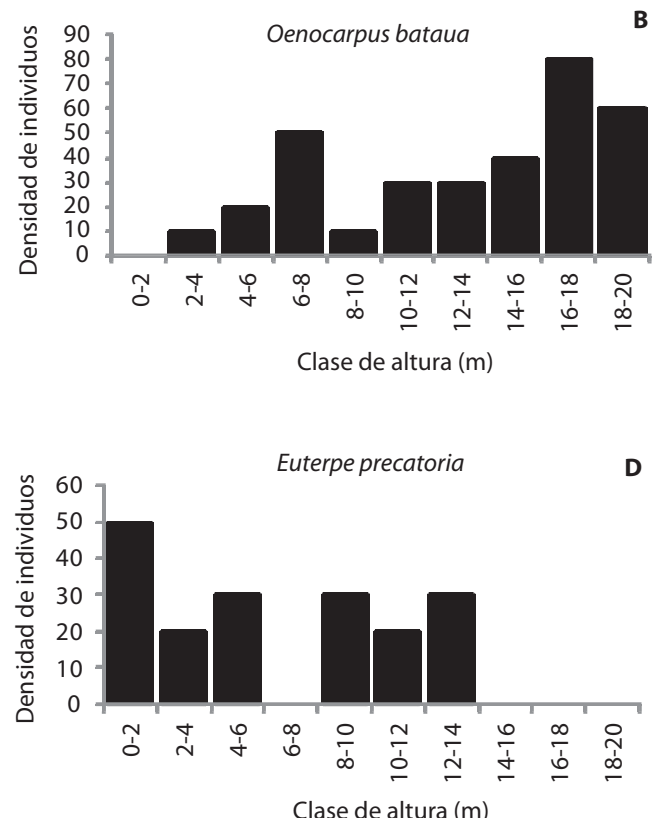

Fig. 5. Abundancia de individuos por clase de altura para (A) todas las palmas; (B) Oenocarpus bataua; (C) Oenocarpus mapora y (D) Euterpe precatoria.

Fig. 5. Steam density per size class of height for (A) all palm species; (B) Oenocarpus bataua y (C) Oenocarpus mapora and (D) Euterpe precatoria.

irregulares (Fig. 5C y D). La estructura en conjunto de todas las palmas, refleja un déficit de individuos de tamaños pequeños que sugiere escasa regeneración natural.

\section{DISCUSIÓN}

Estructura del bosque y riqueza de especies: Los valores de área basal, biomasa aérea y riqueza de especies encontrados para la región de Porce (Colombia), se encuentran dentro de los reportados para otros sitios del neotrópico (Saldarriaga 1991, Kappelle et al. 1996, Kennard 2001, Phillips et al. 2004, King et al. 2005). Se observó que el área basal y la biomasa aérea total por hectárea fueron inversamente proporcionales a la densidad de árboles a través de todo el proceso sucesional estudiado. Este resultado es coherente con el desarrollo de los ecosistemas boscosos a partir del proceso de sucesión secundaria, donde los bosques secundarios jóvenes se caracterizan por presentar mayor densidad de individuos y bajos valores de área basal en relación con los de bosques primarios (Aide et al. 1995, Saldarriaga 1991). Esto se hizo particularmente evidente cuando se analizó la densidad de árboles discriminando por categoría diamétrica. Los bosques sucesionales tempranos (7-11 años) y los intermedios (20-25 años), se caracterizan por presentar densidades de árboles pequeños $(1 \mathrm{~cm} \leq \mathrm{D}<10 \mathrm{~cm})$ más altas, que las que registraron los bosques sucesionales avanzados ( $>40$ años) y los bosques primarios de la zona. En términos de biomasa aérea se encontró que los bosques secundarios tuvieron en promedio una biomasa aérea total dos veces inferior a la de los bosques primarios, con alta variabilidad dentro de ellos $(\mathrm{CV}=57 \%)$. Esto está relacionado con la edad de los sitios y la irregularidad 
en la respuesta y trayectoria sucesional de cada uno, que condicionan la existencia de árboles de ciertos tamaños y especies. Por ejemplo, en el caso de los bosques primarios, las palmas aportaron $5.93 \%$ de la biomasa aérea total del bosque, mientras que en los bosques secundarios este aporte fue sólo de $0.71 \%$. Incluso, fue evidente que la palma Oenocarpus bataua es típica indicadora de bosques primarios por cuanto no se encontró en ningún bosque secundario, tal como se había reportado previamente en otros estudios (Orrego \& del Valle 2003). El aporte de esta especie a la biomasa aérea total del bosque fue de $3.58 \%$. Por otro lado, cuando se analizó la biomasa aérea discriminando por categorías diamétricas, se encontró que los árboles de mayores dimensiones $(\mathrm{D} \geq 10 \mathrm{~cm})$ son los que desempeñan un papel importante en el comportamiento de esta característica, y por ende, en el almacenamiento de carbono al nivel de bosque, especialmente en los estadios sucesionales avanzados y los bosques primarios. Resultados similares se han registrado en La Selva (Costa Rica), donde la biomasa de un árbol de $150 \mathrm{~cm}$ fue aproximadamente igual a la de 607 árboles de $10 \mathrm{~cm}$ de diámetro, y en general, los individuos más grandes constituyeron cerca de $2 \%$ del total, y contribuyeron $23 \%$ en el área basal y $27 \%$ en la biomasa aérea (Clark \& Clark 1996). Por su parte, en Barro Colorado (Panamá) los árboles grandes aportaron entre 35 y $40 \%$ de la biomasa aérea total (Carey et al. 1994). Esta relación insinúa que la pérdida o persistencia de árboles grandes, afecta sustancialmente la cantidad de biomasa del ecosistema, convirtiéndolos en importantes sumideros o fuentes de carbono (Clark \& Clark 1996). En el caso de los bosques primarios de la Región de Porce, se pudo establecer que los árboles de mayores dimensiones $(\mathrm{D} \geq 70 \mathrm{~cm})$, contribuyeron aproximadamente con $42 \%$ de la biomasa aérea total del bosque. El aporte en la biomasa aérea de los árboles pequeños también es importante, especialmente, en los bosques secundarios tempranos y claros o perturbaciones naturales existentes dentro de bosques sucesionales avanzados y primarios, donde abundan este tipo de individuos. Por este motivo es probable que en algunos estudios realizados en bosques tropicales, donde solo se registran árboles con $\mathrm{D} \geq 10 \mathrm{~cm}$, se haya subestimado el aporte en la biomasa aérea de los árboles pequeños. En el caso de los bosques sucesionales de 7-11 años, por ejemplo, los árboles con $1 \mathrm{~cm} \leq \mathrm{D}<10 \mathrm{~cm}$ aportaron cerca del $70 \%$ de la biomasa total de este estadio. La riqueza de especies en general fue mayor en los bosques primarios que en los secundarios, siendo estos últimos representados en su mayoría, por especies pioneras características de estados sucesionales tempranos. En los bosques primarios por el contrario, se encontraron especies importantes como A. excelsum, P. cecropiifolia, $J$. copaia y $V$. ferruginea que sólo se presentan ocasionalmente en bosques sucesionales avanzados, al igual que la palma $O$. batana que podría constituir un indicador de bosques primarios en la región por su presencia exclusiva en esta cobertura.

Los análisis también revelaron que las características estructurales evaluadas (área basal, biomasa aérea y riqueza de especies), en general, aumentaron a medida que avanzó el proceso sucesional. Tendencias análogas fueron encontradas para procesos sucesionales en otras regiones del trópico (e.g. Amazonía, bosque Chiquitano boliviano) donde la recuperación de estas tres características estructurales fue notoria (Saldarriaga 1991, Kennard 2001). Sin embargo, aunque en el presente estudio la riqueza de especies aumentó con el avance de la sucesión, únicamente el bosque secundario de 40 años presentó valores cercanos a los presentados por los bosques primarios de la zona. Este resultado puede estar relacionado con los usos previos de la tierra, que en su mayoría correspondieron a ganadería extensiva y agricultura en pequeñas parcelas (Castillo 1998, Orrego \& del Valle 2003, Agudelo \& Restrepo 2004). De acuerdo con otros estudios, cuando los sitios son intervenidos con intensidades bajas o moderadas, y las fuentes semilleras se encuentran cerca, la riqueza de especies se recupera en forma muy rápida (e.g. décadas). Pero conforme aumenta la intensidad con la que se utilizan los terrenos, la riqueza 
de especies se recuperará más lentamente por la compactación del suelo, las limitaciones espaciales de la dispersión de semillas y las posibilidades de que se den disturbios de origen natural como incendios (Uhl et al. 1988, Guariguata \& Ostertag 2001). Este pudiera ser el caso de los bosques de la Región de Porce, donde los altos valores de densidad aparente de los suelos, encontrados en la mayoría de parcelas establecidas en bosques secundarios, reflejan la compactación de los mismos. Los resultados sugieren que la ganadería extensiva en la región, sumada a la fragmentación de los sitios y la matriz circundante de pastizales que en su mayoría rodea a los bosques secundarios, pudieron ser un factor decisivo para que la riqueza de especies fuera baja en los bosques sucesionales respecto a los valores presentados por los bosques primarios (Yepes-Quintero et al. 2007). En pocas palabras, el tiempo y grado de intervención a la que fueron sometidos los sitios antes del proceso sucesional, pudieron incidir y determinar la respuesta, trayectoria, dirección y tiempo que tomó el mismo (Finegan 1996, 1997, Denslow \& Guzmán 2000, Guariguata \& Ostertag 2001).

En cuanto a las características estructurales como el área basal y la biomasa aérea, algunos autores aseguran que luego de alteraciones drásticas en los sitios, pueden pasar hasta cientos de años, antes de que un bosque sucesional llegue a tener las mismas características que un bosque primario cercano (Finegan 1996, 1997, Guariguata \& Ostertag 2001). Por el contrario, cuando el uso previo de la tierra es la agricultura rotativa, las probabilidades de recuperación estructural del bosque son más altas (Saldarriaga et al. 1988, Lamprecht 1990, Ferreira \& Prance 1999). De hecho, En el caso particular de la biomasa aérea de los bosques secundarios, algunos estudios como el realizado en un bosque tropical en México, han demostrado que la duración del uso previo del suelo en ganadería y cultivos de maíz, tuvo un efecto dramático en la respuesta sucesional medida en tasas de acumulación de la biomasa seca. La curva respuesta tuvo la forma exponencial negativa. Cuando el uso del suelo fue solo dos años, el bosque secundario creció $12.8 \mathrm{t} \mathrm{ha}^{-1} \mathrm{año}^{-1}$, pero cuando el uso previo se prolongó por 30 años, la tasa de recuperación bajó a $4.3 \mathrm{t} \mathrm{ha}^{-1} \mathrm{año}^{-1}$ (Hughes et al. 1999). Todo lo anterior, sumado al hecho de que fueron requeridos aproximadamente 43 años de sucesión para que los bosques sucesionales de la Región de Porce recuperaran 47\% del área basal, $41 \%$ de la biomasa aérea y $44 \%$ de la riqueza de especies, respecto a los valores presentados por los bosques primarios, nos hacen suponer la existencia de una sucesión degradada como consecuencia de las prácticas ganaderas extensivas, que se llevaron a cabo en la región durante la época de la colonización española (Castillo 1998), e incluso hasta principios de 1990 cuando se comenzaron a comprar predios para la construcción de la Central Hidroeléctrica Porce II (Agudelo \& Restrepo 2004). Nuestros resultados y los obtenidos en investigaciones anteriores, donde se reportaron compactación de los suelos hasta $30 \mathrm{~cm}$ de profundidad (Lara 2003), gran dominancia de dos especies pioneras típicas de suelos empobrecidos del género Vismia (Vismia baccifera (L.) Triana \& Planch. y Vismia ferruginea Kunth; Toro 2004, Yepes-Quintero et al. 2007), y tasas de acumulación de biomasa menores en los bosques secundarios en relación con la que presentan los bosques primarios $\left(6 \mathrm{t} \mathrm{ha}^{-1} \mathrm{año}^{-1} v s\right.$. $12 \mathrm{t} \mathrm{ha}^{-1} \mathrm{anno}^{-1}$; Orrego \& del Valle 2003, Sierra et al. 2007) dan cuenta de ello.

Estos hallazgos son preocupantes porque de continuar esta tendencia sucesional, o de existir procesos similares en otros lugares de Colombia y el trópico en general, la capacidad de captura de carbono por parte de los bosques secundarios, por ejemplo, no alcanzaría a cumplir con las expectativas que sobre ellos se tiene actualmente para ayudar a mitigar los efectos del Cambio Climático Global debido a que las tasas de acumulación de biomasa son inferiores a lo esperado (Smith et al. 1997, Guariguata \& Ostertag 2001, Müller 2002, ZambranoBarragán \& Cordero 2008, FAO 2009). Adicionalmente, los resultados también sugieren que procesos sucesionales degradados como el descrito, necesitarían la intervención humana para acelerar y dirigir la trayectoria sucesional 
a escenarios similares a los que se presentan en bosques primarios aledaños, por medio de procedimientos como la restauración ecológica o la regeneración natural asistida, que en la mayoría de los casos, son muy costosas (De las Salas 2002, Orrego \& del Valle 2003). Se recomienda que futuras investigaciones incluyan análisis detallados que tengan en cuenta tratamientos específicos de los sitios (e.g. agrupamiento por diferentes historias de uso, dinámicas sociales, etc.), para concluir con mayor precisión si la sucesión se ve o no condicionada por los usos previos del suelo. Estudios de índole social que permitan reconstruir las dinámicas humanas por las cuales se destruyen y recuperan los bosques, así como estudios etnobotánicos, también son importantes y deberían ser desarrollados, para documentar y referenciar mejor los procesos sucesionales en el trópico.

Estructura diamétrica: La estructura total del bosque primario presentó forma de $\mathrm{J}$ invertida, típica de bosques naturales sin signos fuertes de intervención y cuya dinámica sigue los postulados de la sucesión cíclica donde coexisten árboles de diferentes especies y tamaños (Oliver \& Larson 1990). La forma de esta distribución constituye la mejor garantía para la existencia y supervivencia de la comunidad forestal, puesto que los individuos de mayores dimensiones eliminados ocasionalmente, son sustituidos por individuos de las categorías diamétricas inferiores mediante regeneración natural, y por tanto, la estabilidad demográfica del ecosistema se mantiene (Lamprecht 1990). Resultados similares han sido documentados en otras investigaciones realizadas en el neotrópico (Saldarriaga et al. 1988, Killeen et al. 1998, Ferreira \& Prance 1999, Kennard 2001, Castro et al. 2005). Por su parte, los bosques secundarios (7-10 años, 11 años y 20-25 años de edad) presentaron estructuras en forma de campana o unimodales, típicas de rodales coetáneos que no han alcanzado su máximo desarrollo. En estos bosques predomina como fenómeno demográfico la mortalidad al tiempo que los árboles aumentan de tamaño, por tanto, el reclutamiento de nuevos individuos es limitado. Estas características moldean la forma unimodal de la estructura diamétrica. Respecto a los bosques sucesionales avanzados, llama la atención la estructura diamétrica del bosque secundario de 40 años, puesto que al excluir de los análisis a la especie esciófita Virola sebifera, la estructura de este bosque pasó a ser unimodal en lugar de $\mathrm{J}$ invertida como ocurrió cuando se emplearon todos los árboles registrados. Este comportamiento sugiere que en el sitio se presentaron disturbios naturales o antrópicos en el pasado, que estimularon la presencia de especies en su mayoría heliófitas (Jacaranda copaia, Enterolobium schomburgkii, Vochysia ferruginea, y Schefflera morototoni). Sin embargo, la abundante regeneración de $V$. sebifera, también indica que el bosque se está recuperando de estos disturbios, por lo que el cierre del dosel, crea condiciones favorables para el establecimiento de especies tolerantes a la sombra; adicionalmente animales típicos de bosques altos como los diostedé (Agouti paca) y los murciélagos, que regurgitan las semillas traídas de los bosques primarios vecinos, pueden también contribuir con la alta regeneración de esta especie. Caso contrario ocurrió para el bosque secundario con más de 43 años que tuvo estructura unimodal. La composición florística estuvo dominada principalmente por cuatro especies (Oenocarpus mapora, Cordia bicolor, Miconia albicans y Jacaranda copaia) que conforman una estructura vertical muy uniforme, la cual posiblemente ha inhibido la regeneración del sotobosque. Otra característica como la distancia al bosque primario, también pudo incidir en ello. De esta manera, los resultados de este análisis permiten concluir que 40 años de sucesión en la Región de Porce, no son suficientes para que los bosques secundarios tengan estructuras diamétricas similares a la presentada por los bosques primarios cercanos. Posiblemente las prácticas ganaderas afectaron notablemente las características de los suelos, y ello se ve reflejado en esta característica estructural de los bosques secundarios. De hecho, nuestros resultados contrastan con lo encontrado en tierras dedicadas previamente a la agricultura 
de corta y quema de la Amazonia brasileña, donde la estructura diamétrica de los bosques secundarios fue similar a la de los primarios ( $\mathrm{J}$ invertida) sólo 40 años después del abandono de los predios (Ferreira \& Prance 1999).

Estructura para palmas: La estructura por clases de alturas de las palmas, sugirieron fuertes relaciones entre el establecimiento de éstas y los procesos de intervención de los sitios. La estructura irregular que exhiben las tres especies estudiadas, indican que, en algunos casos, las condiciones del sitio favorecen o desfavorecen el establecimiento de los individuos más jóvenes y la llegada posterior al dosel de algunos de ellos. La estructura de la palma mil pesos (Oenocarpus bataua) en particular, muestra dos cohortes, que debieron llegar allí como consecuencia de la apertura de claros en algún momento del tiempo. De acuerdo con estudios anteriores en la misma zona, esta especie se establece exitosamente en el dosel cuando ocurren claros de origen natural o antrópico que estimulan su crecimiento en altura (Restrepo 2003). De esta manera, las dos cohortes evidentes en los histogramas, sugieren la ocurrencia de perturbaciones dentro del bosque en algún momento del tiempo que favorecieron el establecimiento en el dosel de individuos pertenecientes a esta especie. No obstante, el déficit en el número de individuos pequeños observado, indica pocas perspectivas de relevo generacional para esta especie, por lo que, eventualmente, la población podría declinar de no repetirse un ciclo de nueva formación de claros. Finalmente, la forma unimodal de la distribución cuando se mezclaron todas las palmas, sugiere escasa regeneración de ejemplares pequeños para todos los casos. Ello indica que de manera análoga a lo ocurrido para la palma milpesos, posiblemente hubo un momento en el cual las condiciones ambientales in situ fueron favorables para el establecimiento de los individuos de las diferentes especies, y que estas condiciones no han sido frecuentes en épocas recientes. También es posible que la forma de la distribución se deba al tamaño muestral de la población, puesto que para la misma zona de estudio, se han encontrado distribuciones de alturas en forma de $\mathrm{J}$ invertida, con abundante regeneración (Restrepo 2003).

La mayoría de los parámetros estructurales analizados aumentaron proporcionalmente con la edad de los bosques, pero la recuperación de los mismos en más de 43 años de sucesión, tan solo fue aproximadamente $40 \%$. Factores como los usos previos de la tierra, en su mayoría ganadería extensiva y agricultura y, la fragmentación de los sitios, pudieron determinar esta tendencia donde se observa la existencia de una sucesión degradada en términos de área basal, biomasa aérea y riqueza de especies. No obstante, futuras investigaciones deberán incluir análisis detallados donde se tengan en cuenta tratamientos específico de los sitios (e.g. historia de uso del suelo) que permitan concluir con mayor precisión si la sucesión vegetal se ve o no condicionada por los usos previos a los que fueron sometidos los suelo. Dichos análisis podrían basarse en disciplinas como los sistemas de información geográfica y la dendrocronología, que permitirían sustentar mejor los resultados de este y otros estudios, de manera que se puedan inferir y reconstruir las dinámicas humanas por las cuales se destruyen y recuperan los bosques tropicales, y comprender mejor los procesos sucesionales tropicales.

\section{AGRADECIMIENTOS}

Los autores agradecen a la Dirección de Investigaciones de la Universidad Nacional de Colombia, Sede Medellín (DIME) y a la Dirección Nacional de Investigaciones de la Universidad Nacional de Colombia (DINAIN) por la financiación parcial de la investigación a través de los proyectos de investigación: "Sucesión vegetal, diversidad biológica y acumulación de carbono en el cañón del río Porce, Antioquia" y "Recuperación de la Biomasa en el Cañón de Río Porce". A la Universidad Nacional de Colombia, Sede Medellín, y las Empresas Públicas de Medellín (E.S.P.) por el apoyo logístico. A los profesores Álvaro Lema Tapias, María Claudia Díez y Oswaldo Velásquez por su colaboración y asistencia técnica. A Rodrigo 
Caballero, Duván Correa, Eduardo Gutiérrez y Javier Gutiérrez, por su valiosa colaboración en las actividades de campo.

\section{RESUMEN}

Lugares susceptibles a perturbaciones naturales o antrópicas pueden recuperar la cobertura boscosa. La sucesión tropical puede ser afectada por factores tales como perturbaciones, distancia al bosque original, topografía y clima local. Estos factores determinan la composición de especies y la tasa de recuperación de los sitios. Se estudió la sucesión en suelos que habían sido usados para el establecimiento de pasturas ganaderas por varias décadas en la Región Porce de Colombia (bosques Colombianos de los Andes). Se midieron un total de veinticinco parcelas, incluyendo nueve parcelas $(20 \times 50 \mathrm{~m})$ en bosques primarios y dieciséis $(20 \times 25 \mathrm{~m})$ en bosques secundarios. Se midieron todos los árboles con un diámetro $\geq 1.0 \mathrm{~cm}$. Se analizó la densidad, área basal, biomasa aérea y riqueza de especies, en un bosque con un proceso sucesional de ca. 43 años y en un bosque primario. Las edades de los bosques secundarios fueron obtenidas en estudios previos usando la datación con carbono catorce $\left(\mathrm{C}^{14}\right)$, fotografías aéreas y el análisis de imágenes de satélite de alta resolución (entre 7 y $>43$ años). En total, 1143 y 1766 individuos se midieron en bosque primario y secundario, respectivamente. El área basal $\left(5.7\right.$ a $\left.85.4 \mathrm{~m}^{2} \mathrm{ha}^{-1}\right)$, la biomasa aérea (19.1 a 1011.5 $\mathrm{t} \mathrm{ha}^{-1}$ ) y la riqueza de especies (4 a 69) aumentaron directamente con la edad de los sitios, mientras que la densidad de árboles disminuyó (3 180 a 590). Las distribuciones diamétricas fueron en J-invertida para los bosques primarios y unimodal para los secundarios. Tres especies de palmas fueron abundantes y exclusivas de bosques secundarios viejos y bosques primarios: Oenocarpus mapora, Euterpe precatoria y Oenocarpus bataua. Cohortes de estas palmas aparecieron después de perturbaciones del bosque. La recuperación de la estructura de los bosques secundarios en más de 43 años de sucesión fue $40 \%$ e indica que varios factores están interactuando y afectando la sucesión de los bosques en el área (e.g. agricultura, ganadería extensiva, minería, etc.).

Palabras clave: biomasa aérea, bosques primarios tropicales, bosques secundarios tropicales, estructura diamétrica, sucesión vegetal.

\section{REFERENCIAS}

Aide, T.M., J.K. Zimmerman, L. Herrera \& M. Rosario. 1995. Forest recovery in

abandoned tropical pastures in Puerto Rico. Forest. Ecol. Manag. 77: 77-86.
Agudelo, E.A. \& J.C. Restrepo. 2004. Análisis multitemporal de las coberturas en áreas del proyecto hidroeléctrico Porce II. Trabajo de grado Ingeniería Forestal, Universidad Nacional de Colombia, Medellín, Colombia.

Brown, S. \& A.E. Lugo. 1990. Tropical secondary forest. J. Trop. Ecol. 6: 1-32.

Brown, S. 1997. Estimating biomass and biomass change of tropical forest: a primer. FAO Forestry Paper 134, FAO, Roma, Italia.

Carey, E.V., S. Brown, A.J.R. Gillespie \& A.E. Lugo. 1994. Tree mortality in mature lowland tropical moist and tropical lower montane moist forest of Venezuela. Biotropica 26: 255-265.

Castillo, N. 1998. Los antiguos pobladores del valle medio del río Porce: aproximación inicial desde el estudio arqueológico del proyecto Porce II. Universidad de Antioquia, Medellín, Colombia.

Castro, G., R. Nygard, B. Gonzales \& P.C. Oden. 2005. Stand dynamics and basal area change in a tropical dry forest reserve in Nicaragua. Forest. Ecol. Manag. 208: 63-75.

Clark, D.B. \& D.A. Clark. 1996. Abundance, growth and mortality of very large trees in Neotropical lowland rain forest. Forest. Ecol. Manag. 80: 235-244.

Clark, D.A. 2007. Detecting tropical forests responses to global climatic and atmospheric change: current challenges and a way forward. Biotropica 39: 4-19.

Denslow, J.S. \& S. Guzman. 2000. Variation in stand structure, light, and seedling abundance across a tropical moist forest chronosequence, Panama. J. Veg. Sci. 11: 201-212.

De las Salas, G. 2002. Los bosques secundarios de América tropical: perspectivas para su manejo sostenible. Bois Forêts. Trop. 272: 63-73.

De Walt, S.J., S.K. Maliakal \& J.S. Denslow. 2003. Changes in vegetation structure and composition along a tropical forest chronosequences: implications for wildlife. Forest. Ecol. Manag. 6227: 1-13.

Ewel, J. 1980. Tropical succession: manifold routes to maturity. Biotropica 12: 2-7.

FAO-Organización de las Naciones Unidas para la Agricultura y la Alimentación. 2009. Situación de los bosques en el mundo 2009. Grupo de Ventas y Comercialización, División de Comunicación. Roma, Italia. 
Ferreira, L.V. \& G.T. Prance. 1999. Ecosystem recovery in terra firme forest after cutting and burning: a comparison on species richness, floristic composition and forest structure in the Jaú National Park, Amazonia. Bot. J. Linn. Soc. 130: 97-110.

Finegan, B. 1996. Pattern and process in neotropical secondary rain forest: the first 100 years of succession. Trends. Ecol. Evol. 11: 119-124.

Finegan, B. 1997. Bases ecológicas para el manejo de bosques secundarios de las zonas húmedas del trópico americano, recuperación de la biodiversidad y producción sostenible de madera, p. 106-127. In Deutzche Gesellshaft für Technische Zusammenarbeit (GTZ) GMBH, Ministerio de Cooperación Técnica del Reino de los Países Bajos \& IKC Natuur Beheer National Reference Center for Nature Management (eds.).Taller internacional sobre el estado actual y potencial de manejo y desarrollo del bosque secundario tropical en América Latina. Secretaría de Tempore Venezuela (TCA), Consejo Centroamericano de Bosques y Áreas Protegidas. Pucallpa, Perú.

Guariguata, M.R. \& R. Ostertag. 2001. Neotropical secondary forest successions: changes in structural and functional characteristics. Forest. Ecol. Manag.148: 185-206.

Hua, Q. \& M. Barbetti. 2004. Review of troposferic bomb ${ }^{14} \mathrm{C}$ data for carbon cycle for modeling and age calibration purposes. Radiocarbon 46: 1273-1298.

Hughes, R.F., J.F. Kauffman \& V.V. Jaramillo. 1999. Biomass, carbon, and nutrient dynamics of secondary forests in a humid tropical region of Mexico. Ecology 80: 1982-1907.

Jaramillo, D. 1989. Estudio general de suelos, erosión y uso potencial agropecuario para los proyectos hidroeléctricos Porce II y Porce III. Empresas Públicas de Medellín. Unidad de Planeación, Estudios de Impacto Ambiental. Medellín, Colombia.

Kappelle, M., M. Geuze, M. Leal \& A.M. Cleef. 1996. Successional age and forest structure in a Costa Rican upper montane Quercus forest. J. Trop. Ecol. 15: 143-157.

Kennard, K.K. 2001. Secondary forest succession in a tropical dry forest: patterns of development across a 50-year chronosequence in lowland Bolivia. J. Trop. Ecol. 18: 53-66.

Killeen, T.J., A. Jarmin, F. Mamani \& N. Rojas. 1998. Diversity, composition and structure of tropical semideciduous forest in the Chiquitanía region of Santa Cruz, Bolivia. J. Trop. Ecol. 14: 803-827.
King, D.A., S. Davies \& N.S.M. Noor. 2005. Growth and mortality are related to adult tree size in a Malaysian mixed Dipterocarp forest. Forest. Ecol. Manag. 223: 152-158.

Lamprecht, H. 1990. Silvicultura en los trópicos: Los ecosistemas forestales en los bosques tropicales y sus especies arbóreas-posibilidades y métodos para un aprovechamiento sostenido. (GTZ) GMBH, Eschborn, Alemania.

Lara, W. 2003. Estudio edafológico del carbono en distintas coberturas vegetales de la región de Porce, Colombia. Trabajo de grado Ingeniería Forestal, Universidad Nacional de Colombia, Medellín, Colombia.

Márquez, L. \& A. Roy. 2000. Elementos técnicos para inventarios de carbono en uso del suelo. Fundación solar, Ciudad de Guatemala, Guatemala.

Moreno, F.H. 2004. Soil carbon dynamics in primary and secondary tropical forest in Colombia. Thesis Doctor of Philosophy in Biology, Florida International University, Miami, Florida.

McDicken, K.G. 1997. A guide to monitoring carbon storage in forestry and agroforestry projects. Winrock International Institute for Agricultural Development, Forest Carbon Monitoring Program,Virginia, EEUU.

Müller, E. 2002. Los bosques secundarios salen a la luz. OIMT Act. Fores. Trop. 10: 16-18.

Oliver, C. \& B.C. Larson. 1990. Forest stands dynamics. McGraw Hill, Nueva York, Nueva York, EEUU.

Orrego, S.A \& J.I. del Valle. 2003. Existencias y tasas de incremento neto de la biomasa y del carbono en bosques primarios intervenidos y secundarios, p. 215-242. In S.A. Orrego, J.I. del Valle \& F.H. Moreno (eds.). Medición de captura de carbono en ecosistemas forestales tropicales de Colombia: contribuciones para la mitigación del cambio climático. Universidad Nacional de Colombia sede Medellín, Centro Andino para la Economía en el Medio Ambiente, Bogotá, Colombia.

Phillips O.L. \& A.H. Gentry. 1994. Increasing turnover through time in tropical forest. Science 263: 954958.

Phillips O.L., T. Baker, L. Arroyo, N. Higuchi, T. Killeen, W.F. Laurance, S.L. Lewis, J. Lloyd, Y. Malhi, A. Monteagudo, D. Neill, P. Nuñez, N. Silva, J. Terborgh, R. Vásquez, M. Alexiades, S. Almeida, S. Brown, J. Chave, J.A. Comiskey, C.I. Czimczik, A. Di Fiore, T. Erwin, C. Kuebler, S.G. Laurance, H.E.M. Nascimento, J. Olivier, W. Palacios, S. Patiño, N. Pitman, C.A. Quesada, M. Saldias, A. Torres 
\& B. Vicenti. 2004. Pattern and process in Amazon tree turnover, 1976-2001. Philos. T. Roy. Soc. B. 1438: 1-27.

Reimer, P.J., T.A. Brown \& R.W. Reimer. 2004. Discussion: Reporting and calibration of Post-Bomb ${ }^{14} \mathrm{C}$ data. Radiocarbon 46: 1299-1304.

Restrepo, D.E. 2003. Biomasa de la comunidad de palmas en bosques primarios de la cuenca media del río Porce. Trabajo de grado Ingeniería Forestal, Universidad Nacional de Colombia, Medellín, Colombia.

Rollet, B. 1980. El bosque tropical y la biosfera, p. 36-67. In Unesco/PNUMA/FAO (eds.). Ecosistemas de bosques tropicales: informe sobre el estado de conocimientos. Unesco-CIFCA, París, Francia.

Ricker, M. \& D. Daly. 1998. Botánica económica en bosques tropicales: principios y métodos para su estudio y aprovechamiento. Editorial Diana, México, D.F, México.

Saldarriaga, J.G. 1991. Estudios en la Amazonía colombiana V: Recuperación de la Selva de "Tierra Firme" en el alto río Negro Amazonía colombiana-venezolana. TROPENBOS Colombia, Bogotá, Colombia.

Saldarriaga, J.G., D.C. West, M.L. Therp \& C. Uhl. 1988. Long-term chronosequence of forest succession in the upper Rio Negro of Colombia and Venezuela. J. Ecol. 76: 938-958.

Sierra, C.A., J.I. del Valle, S.A. Orrego, F.H. Moreno, M.A. Harmon, M. Zapata, G.J. Colorado, M.A. Herrera, W. Lara, D.E. Restrepo, L.M. Berrouet, L.M. Loaiza, J.F. Benjumea. 2007. Total carbon stocks in a tropical forest landscape of the Porce region, Colombia. Forest. Ecol. Manag. 243: 299-309.

Silvertown, J.W. \& J. Lovett Doust. 1993. Introduction to plant population biology. Blackwell Science, Oxford, Inglaterra.

Smith, J., C. Sabogal, W. de Jong \& D. Kaimowitz. 1997. Bosques secundarios como recurso para el desarrollo rural y la conservación ambiental en los trópicos de América Latina. Center for International Forestry Research (CIFOR), Occasional Paper No. 13. Jakarta, Indonesia.

Sprent, P. 1989. Applied nonparametric statistical methods. Chapman \& Hall, England.

Toro, J.C. 2004. Sucesión temprana tropical en pasturas abandonadas y rastrojos bajos: biodiversidad y productividad. Trabajo de grado Ingeniería Forestal, Universidad Nacional de Colombia, Medellín, Colombia.

Uhl, C., R. Buschbacher \& E.A.S. Serrao. 1988. Abandoned pastures in eastern Amazonia. I. Patterns of plan succession. J. Ecol. 76: 663-681.

Walpole, R., \& R. Myers 1987. Probabilidad y estadística para ingenieros. Interamericana, México D.F, México.

Worbes, M. 1989. Dating tropical trees by means of C-14 bomb test. Ecology 70:503-507.

Yepes-Quintero, A.P., S.L. Jaramillo-Restrepo, J.I. del Valle-Arango, S.A. Orrego-Suáza. 2007. Diversidad y composición florística en bosques sucesionales andinos de la región del río Porce, Colombia. Revista de Actualidades Biológicas 29: 103-113.

Zambrano-Barragán, C. \& D. Cordero. 2008. REDD en América del REDD: Experiencias y herramientas útiles. Unión Internacional para la Conservación de la Naturaleza (UICN), Quito, Ecuador.

Zapata, M., J.I. del Valle, S.A. Orrego \& F.H. Moreno. 2003. Estimación de la biomasa, p. 8-44. In C.A. Sierra \& M. Zapata (eds.). Formulación del plan de manejo de la vegetación en el área de influencia de la central hidroeléctrica Porce III, Contrato 3/DJ/1367/17-Acta No. 28, Universidad Nacional de Colombia, Sede Medellín-Empresas Públicas de Medellín E. S. P. Departamento de Ciencias Forestales, Universidad Nacional de Colombia. Medellín, Colombia. 
